\title{
Processes of mixture formation, ignition and combustion of a diesel engine
}

\author{
Jumaniyoz Ismatov ${ }^{1 *}$, and Obidjon Ergashev ${ }^{2}$ \\ ${ }^{1}$ Tashkent State Technical University named after Islam Karimov, Tashkent, Uzbekistan \\ ${ }^{2}$ Tashkent State Transport University, Tashkent, Uzbekistan
}

\begin{abstract}
One of the most significant concepts of deep restructuring of the economic mechanism of the Republic of Uzbekistan is the creation of an integral, effective and flexible system of economic management and the implementation on this basis of a program to improve the technical level and quality of machines. The car park of our country is more replenished with vehicles with diesel engines. The development of contemporary diesel engine building is proceeding by forcing engines: increasing the average effective pressure and speed. Therefore, high reliability and service life, fuel efficiency and environmental performance are the basic criteria for their quality. Forcing diesel engines leads to an increase in thermal and mechanical loads on the basic parts of the cylinder-piston group (CPG) (piston, liner and cylinder head), a significant increase in their temperature, as well as the temperature of piston rings and valves. Overheating of parts is accompanied by the formation of temperature fields with a pronounced uneven temperature distribution and, as a result, an increase in thermal stresses with a simultaneous deterioration of the mechanical properties of the material, the formation of cracks and, finally, the destruction of the part. In this regard, the task of protecting parts from excessive exposure to high thermal loads from the working fluid becomes urgent, or, in other words, the task of creating a diesel engine with reduced heat removal from the working fluid.
\end{abstract}

\section{Introduction}

First of all, with the provision of contemporary environmental requirements, the creation of a highly efficient engine with reduced heat removal from the working fluid is associated with the solution of a number of other problems. First of all, this refers to the reduction of nitrogen oxides in combustion products while simultaneously reducing the specific fuel consumption.

Taken together, these tasks constitute a major scientific and technical problem, which has significant industrial and economic importance, since its solution makes it possible to increase the scientific and technical level of design and creation of new

* Corresponding author: jumaniyoz132@mail.ru 
promising engines and to obtain an economic effect by reducing the cost and costs of experimental debugging of diesel engines with reduced thermal losses.

The processes of fuel supply, mixture formation, ignition and combustion are characterized by extreme unsteadiness. Due to the movement of the piston, as well as due to the intense heat release after ignition, the pressure and temperature in the cylinder are constantly changing. The fuel is injected at variable pressure, and the formed spray of atomized fuel, non-uniform in structure, interacts with an air vortex organized in the chamber. The complexity of the processes is aggravated by the wide fractional composition of fuels. The complexity of the conditions accompanying mixture formation, ignition and combustion of fuel does not allow a complete description of the mechanisms of physicochemical processes occurring in a diesel cylinder. However, on the basis of many years of experience gained in the creation of high-speed diesel engines, as well as a large number of works by domestic and foreign researchers on the study of the processes of mixture formation, ignition and combustion, a number of provisions can be formulated about the processes occurring in the diesel cylinder.

\section{Materials and Methods}

In basic periods of the combustion process, fuel does not burn immediately upon entering the diesel cylinder. Mixture formation, ignition and combustion are complex physicochemical processes continuously developing in time and volume of the combustion chamber, which can be conditionally divided into five periods based on the analysis of indicator diagrams (Figure 1).

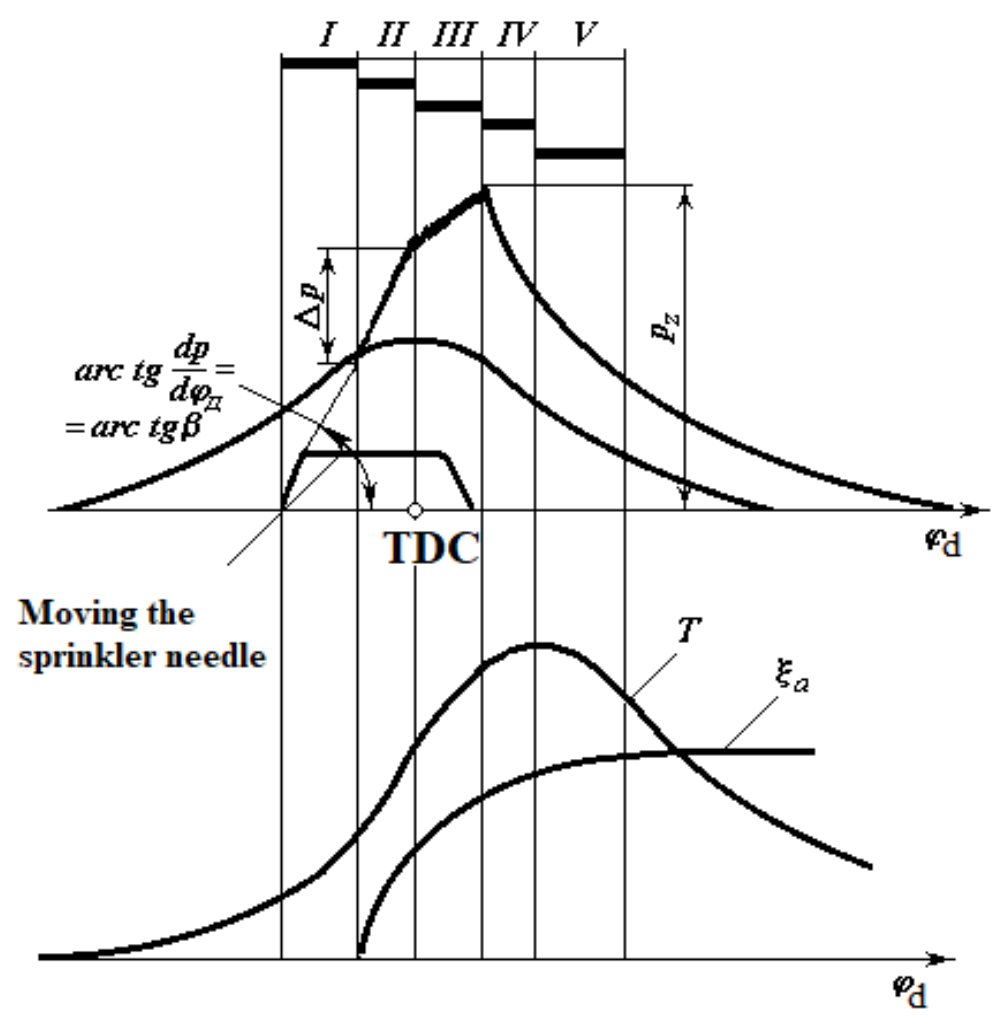

Fig.1. Diesel indicator diagram 
Such a division of the combustion process into phases is somewhat different from the previously accepted one. However, it is more expedient since it reflects all possible types of processes in real engines. A.I. Tolstov [1] in the four-period scheme of the process does not distinguish the second period, although it is very important in the analysis of parameters that assess the rigidity of the combustion process. W.V. Iost [2] there is no main combustion period, the end of which determines the timeliness of heat release, and it is advisable to establish this period when analyzing the efficiency of the operating cycle. G.R. Ricardo [3], characterizing the process in diesel engines, indicates only the first three phases of the process, although in many cases, especially in supercharged diesel engines, a significant part of the heat is released after reaching the maximum pressure in the cycle. In the studies of D.D. Brose [4], three periods are also noted, while the third continues until the end of the afterburning, which does not allow analyzing the phase of the main combustion.

\section{Results and Discussion}

The induction period (ignition lag or ignition delay) (I) lasts from the beginning of the flow of fuel into the diesel cylinder until the moment of visible combustion (the moment corresponding to the separation of the combustion line on the indicator diagram from the compression line). The period of rapid (explosive) combustion (II) is characterized by a sharp increase in pressure in the diesel cylinder. The controlled combustion period (III) lasts from the end of the second period until the maximum pressure is reached. The period of the main combustion (IV) corresponds to the time from the moment of reaching the maximum pressure to the moment at which the highest temperature of the cycle is noted. The delayed combustion period $(\mathrm{V})$ is defined as the time from the moment the maximum temperature is reached until the near end of combustion.

Depending on the type of engine, its mode of operation, the scheme of the organization of the workflow, the nature of heat release may change. In some cases, it is not possible to clearly distinguish individual periods of the combustion process, which confirms the conventionality of dividing the combustion process into separate stages.

Induction period, even R. Diesel believed that the process of oxidation and ignition of fuel is preceded by its evaporation. Subsequent studies by Wollers and Emke and theoretical ones by K. Neumann shaken, but not for long, these opinions. After the work of Rothrock and Waldron, published in 1932, in which, based on direct observation, it was concluded that a significant part of the fuel supplied to the cylinder during the induction period before ignition began, the latter point of view, confirmed by numerous studies, became prevailing. This position is also consistent with the fact that the rate of hydrocarbon oxidation reactions in the liquid phase, which have a relatively low temperature, due to insufficient molecular energy and low intensity of the formation of active reaction centers, and also due to the limiting role of oxygen diffusion into liquid fuel, is significantly lower than observed in a diesel cylinder. In addition, the activation energy of the initial oxidation reaction is greater than the heat of vaporization.

Physical model of the fuel ignition process, as it enters the engine cylinder, the fuel is crushed into droplets, and part of it can settle on the wall of the combustion chamber, forming a complex wall structure with film elements. After heating, fuel vaporization begins from the surface of the droplets in the volume of the jet and the region of the nearwall layer, as a result of which a vapor-air mixture is formed with a continuously varying concentration both in the time of development of the process and in the volume of the combustion chamber due to the processes of molecular diffusion and transfer of fuel vapors. The formation of a vapor-air mixture takes place in a space with temperatures continuously varying in different zones of the combustion chamber due to the compression 
of the working mixture, heat exchange between fuel and air or the surface of the combustion chamber, as well as fuel evaporation. Ignition will occur at the point of the combustion chamber at the moment when there is a combination of the required concentration of the mixture with the corresponding autoignition temperature.

Kinetics of chemical reactions of hydrocarbon oxidation [5], The slow oxidation of hydrocarbons in a gaseous medium, which takes place in a diesel cylinder during the induction period, is a complex multistage process, during which, when the fuel is heated and evaporated, extremely unstable intermediate formations of molecular and radical types are obtained, the interaction of which determines the course of the reaction. Therefore, the theory of hydrocarbon oxidation is still far from the stage when it is possible to accurately predict the rate and direction of reactions under given conditions, based on the structural and chemical structure of the fuel [6].

Based on the work of scientists in physical chemistry, who developed the theory of chain reactions of oxidative processes, the following model of multistage initial oxidation (self-ignition) of fuel in a diesel cylinder has been formulated. During the induction period, the fuel evaporates and, according to the unbranched chain reaction scheme, the products of incomplete oxidation are formed - aldehydes, in particular formaldehydes, which is accompanied by the luminescent glow of the entire charge. Further, the process develops according to the scheme of a degenerate chain reaction with the formation of peroxides and radicals, which give a secondary cold flame, spreading over the volume of the charge.

After accumulating the required number of active reaction centers with the simultaneous release of heat, thermal acceleration of the reaction occurs, leading to explosive combustion with hot yellow flame in the reaction zone of the combustible mixture. Such a mechanism of hydrocarbon oxidation can occur at elevated pressures $(2-3 \mathrm{MPa})$ and at a temperature of $500-700{ }^{0} \mathrm{C}$, which corresponds to the conditions of ignition of the mixture in diesel cylinders [7]. The specific kinetic mechanism can vary significantly depending on the chemical and group composition of the hydrocarbons included in the fuel.

The duration of the induction period $\tau_{\mathrm{i}}$ largely determine the course of the subsequent stages of the process. The main factors that determine the duration of $\tau_{\mathrm{i}}$ ) the structural composition of the fuel and the thermodynamic parameters of the air charge. An approximate estimate of the induction period can be carried out by the formula [22].

$$
\tau_{i}=B \cdot 10^{-2} \sqrt{C} \sqrt{\frac{T_{k}}{p_{k} \exp \left(\frac{E C^{0,34}}{R T_{k}}\right)}} .
$$

Here $B=2 \cdot 10^{-4}\left(1 \ldots 1,6 \cdot 10^{-4} n_{\partial}\right) ; C=\left\lfloor 1+0,5 \delta\left(V_{s} / V_{s_{1}}\right)(\varepsilon-1)\right\rfloor / \varepsilon$,

Where $\delta=\left[(1+\lambda / 4)-\left(\cos \theta_{\partial}+\lambda \cdot \cos 2 \theta_{\partial} / 4\right)\right] ; V_{s_{1}}$ is the working volume of the cylinder at the moment of closing the distribution bodies.

The duration of the $\tau_{i}$ period depends little on the parameters of the injection process. The fuel jet will always contain droplets of various sizes, including droplets of the optimal size for the given conditions of mixture formation [3]. To a greater extent, $\tau_{i}$ can be influenced by the direction and range of the fuel jets, which can fall into zones with different temperatures and different chamber surface parts.

The rapid combustion period is characterized by the rapid development of the process with high rates of fuel burn up and an intense rise in temperature and pressure. The combustion rate increases almost instantly from almost zero to the maximum (Fig. 2.) and usually remains throughout the entire period. This determines the rigidity of the combustion process - the level of dynamic loads acting on the parts of the engine crank mechanism. 


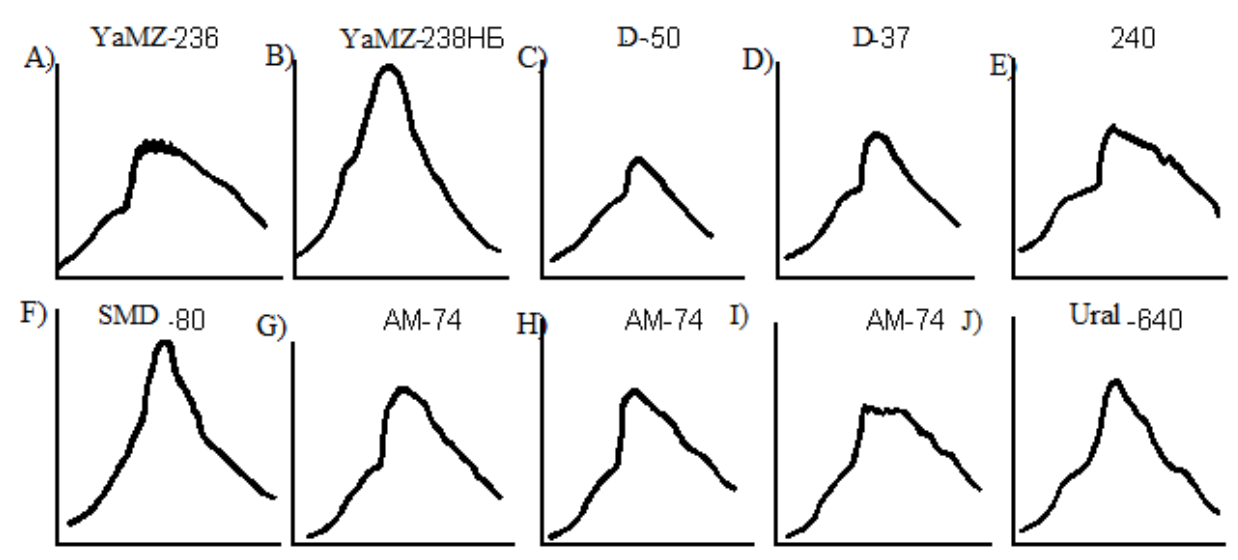

Fig. 2. Indicator diagrams of tractor and automobile diesel engines: $a, d, g, h$ - direct fuel injection; $b, f$ - supercharged; $b, f$, - with vortex chambers; $e, h$ - with a camera of the CRDI type; $j$ - with a chamber with wall mixture formation

The period of rapid combustion is observed in most modern diesel engines, including diesel engines with direct fuel injection (Fig. 2, a, d, g, h), diesel engines with supercharging (Fig. 2, b, f), engines with vortex chambers ( Fig. 2, c) and even engines with volumetric wall (CRDI type) (Fig. 2, e, h) and wall (film) chambers (Fig. 2, j). This period for all types of chambers and mixture formation schemes is explained by the fact that no effective ways of eliminating it have been found without deteriorating fuel efficiency and exhaust smoke.

As shown by many years of operating experience, the intensity of wear and service life, the level of sound and the toxicity of diesel exhaust gases largely depend on the severity of combustion. The increased hardness of combustion limits fuel use with a light fractional composition and alternative fuels in diesel engines.

The current state of the theory of wear resistance of machine parts does not allow predicting their service life based on the forces $\mathrm{P}$ acting on them and, moreover, the speed of their application. The breakdown of oil films between the contacting surfaces due to shocks arising from vibrations of engine parts also contributes to increased wear. If the period $\mathrm{T}_{0}$ of free vibrations of the system is commensurate with the duration of the force application, then the dynamic component $\Delta y$ of static displacement can be expressed by the dependence [8]:

$$
\Delta y=\frac{T_{0}}{2 c} \frac{d P}{d t}
$$




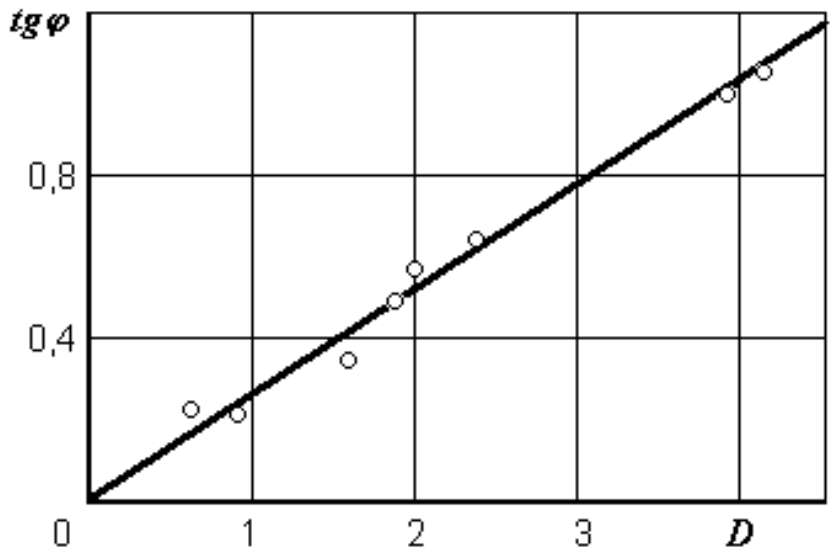

Fig.3. Dependence of $\operatorname{tg} \varphi$ of the wear curve on the dynamic factor $D$

These phenomena as applied to ATD are confirmed by studies of vibrations of the piston ring of a single-cylinder diesel engine CHR-2022, arising during the period of an intensive increase in pressure [9].

To assess the severity of combustion, indicators such as the average $(d p / d \varphi)^{c p}$ or the maximum rate of pressure rise $(d p / d \varphi)^{\max }$ are usually used, as well as the maximum cycle pressure and the degree of pressure increase $\lambda$ (see fig. 1). However, these parameters are not perfect enough since they do not assess the effect of hard combustion on the resulting vibrations of parts and, accordingly, on the wear rate and sound level (fig. 3). According to the mathematical interpretation, oscillations are a consequence of applying the variable harmonic components of the pressure change function in the diesel cylinders to the parts. In the frequency range $500-2000 \mathrm{~Hz}$, these amplitudes increase with an increase in the rate of rising of pressure and the value of $p_{z}$ which determines the increase in pressures $\Delta p$ during the period of rapid combustion. It is advisable to assess the degree of dynamism of the indicator diagrams by an indicator that considers both the steepness of the pressure rise front and the duration of the section. As such, an indicator can be used a dynamic factor determined by the formula [9].

$$
D=\left(\frac{d p}{d \varphi}\right)^{\max } \Delta p
$$

The nature of heat release during the controlled combustion period is largely determined by the speed of the fuel supplied to the cylinder. During this period, the fuel supplied during the induction period and did not manage to burn during the second period and a significant part of the fuel injected during the third period burns. Fuel combustion in the third period occurs at a high total excess air ratio, and the processes of mixing air with fuel do not limit the reaction rate. Therefore, the turbulization of the charge has little effect on the rate of heat release in this period. The nature of the propagation of jets in the volume of the combustion chamber and the dispersion of the atomized fuel has a great influence on the course of the reaction. The duration of the period increases with an increase in $V_{c}$ and an increase in injection pressure.

Ignition of the fuel entering the cylinder during this period occurs according to a onestage process, according to which, at high temperatures, the breaking of intramolecular bonds in the fuel and the formation of active radicals with free valence, which can directly 
interact with an oxygen molecule, become possible. Along with the rapid evaporation of fuel, the process of flame propagation from the ignition sources to the adjacent zones of the combustion chamber occurs.

In the third period, an intensive formation of reaction products is observed in various parts of the combustion chamber. It is possible to release free carbon in over-enriched, flame-engulfed zones with a high temperature, which does not have time to completely burn out under conditions of local air deficiency in subsequent periods.

The main combustion period is characterized by the propagation of flame from the formed combustion zones throughout the entire space of the combustion chamber with the achievement of a high volumetric flow rate of the processes. By the end of the period, the excess air ratio and the number of intermediate reaction products sharply decrease, and the amount of the final products of fuel oxidation increases. In this case, the temperature of the mixture reaches its maximum value. Depending on the engine load, the period of the main combustion can end after $10-35^{\circ}$ PKV after TDC. Since the change in pressure in this period no longer determines the dynamic intensity of the cycle, and the earlier release of heat contributes to the improvement of the efficiency of the cycle, it is necessary to strive for the maximum intensification of the combustion process in this period and the achievement of the highest temperature at the moment as close as possible to TDC.

The combustion process is turbulent-diffusional, i.e. the intensity of the reactions is determined by the rate of mutual diffusion of fuel and oxidant molecules and by the processes of transfer and mixing of matter. Physical rather than chemical processes play the main role in the combustion process.

The turbulence of the charge and the preservation of the vortex motion of the air in the combustion chamber are of paramount importance and determine the dynamics of the oxidation processes.

The period of delayed combustion (afterburning) is observed after the end of the main phase of fuel combustion and extends to a significant part of the expansion stroke. At full load, afterburning can last even before the valves are opened. The presence of an afterburning period worsens the economic performance of a diesel engine, although the amount of heat released during this period may be small.

The afterburning process is of a diffusion nature, and if it cannot be avoided, then for the fastest completion of the afterburning, it is necessary to ensure that the processes of transfer and mixing of the products of incomplete oxidation of fuel and air oxygen are preserved in the engine cylinder. The fulfillment of this condition is all the more necessary because the afterburning process is characterized by a very low average and local coefficients of excess air.

Requirements for the organization of an optimal heat release process. The main task of mixture formation is to organize the optimal heat release in the diesel cylinder, which ensures the maximum possible efficiency with the admissible dynamics of heat release in the initial period of the combustion process, which determines the durability of the diesel engine and the noise from its operation.

The relationship between heat release and efficiency dynamics can be established based on the analysis of the thermodynamic cycle of a diesel engine. In the classical theory, three types of cycles of internal combustion engines are distinguished (Fig. 4): Otto cycle with heat supply at constant pressure; the Sabate-Trinkler cycle, in which part of the heat is supplied at a constant volume and a part of the heat at a constant pressure. Such a formalization of cycles is very arbitrary since it does not fully reflect the real processes taking place in engines. In reality, heat release occurs at a simultaneously changing volume and pressure of the working fluid. 

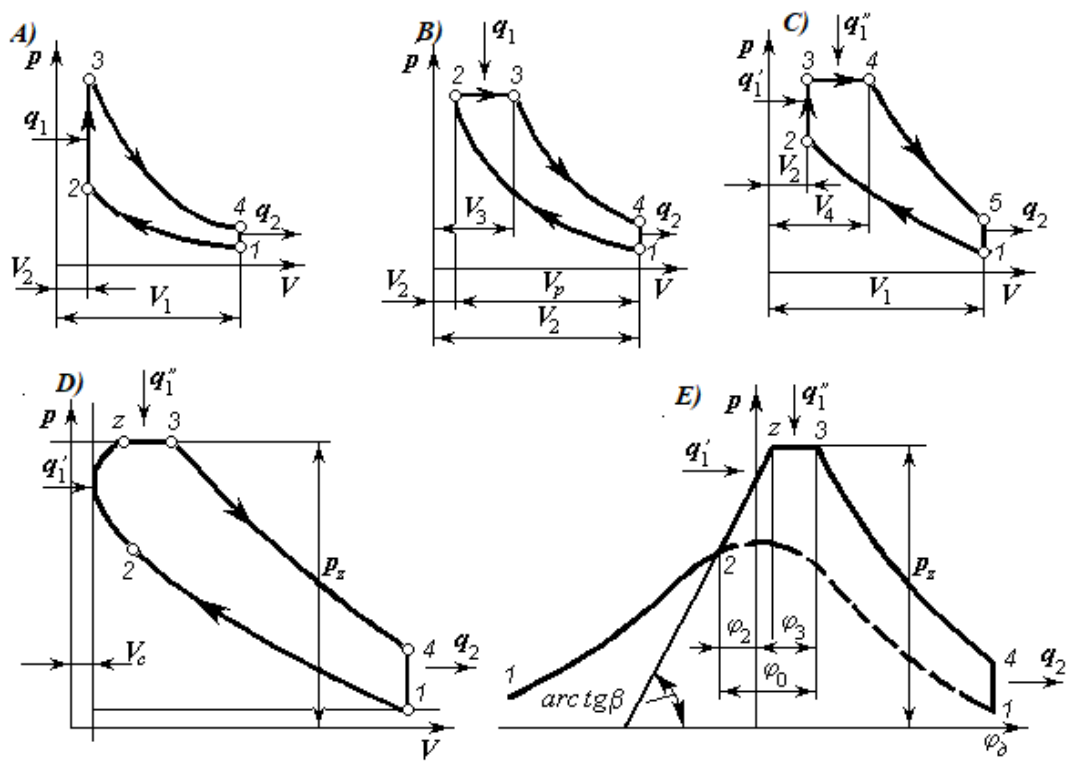

Fig.4. Diagrams of Otto's thermodynamic cycles (a); diesel engine (b); Sabate - Trinkler (c) and cycles limited by the rate of heat release $(d, e)$

As a thermodynamic cycle of a high-speed engine with compression ignition, it is advisable to take a cycle in which the heat supply process is also regulated by the specified limits of the intensity of the pressure rise $\beta=d p / d \varphi$ and maximum pressures $p_{z}$.

The cycle provides for the beginning of the supply of heat before the piston approaches the TDC (see Fig. 4), while the pressure increases at a constant rate until reaching the maximum values. The second stage of heat supply is carried out at $p_{z}=$ const. This course of the cycle provides the best economic performance for the given dependencies [10]:

$$
\begin{gathered}
\eta_{m . \varkappa}=1-\frac{A}{(k-1) Q_{3}} \cdot \frac{p_{z}\left[v_{c}+a\left(\varphi_{0}-\varphi_{2}\right)^{2}\right]^{k}}{\left(v_{c} \varepsilon\right)^{k-1}-R T} ; \\
\varphi_{0}-\varphi_{2}=\sqrt{\varphi_{2}^{2}-A} ; \quad A=\left(p_{z}-p_{2}\right) \cdot\left(\frac{2 \varphi_{2}}{\beta}+\frac{\varphi_{2}^{2}}{k p_{z}}+\frac{v_{c}}{p_{z} k a}-\frac{2 p_{z} \varphi_{2}}{p_{z} \beta}\right)+ \\
+\left(p_{z}-p_{2}\right)^{2} \cdot\left(\frac{p_{2}}{p_{z} \beta^{2}}-\frac{\varphi_{2}}{p_{z} \beta}-\frac{1}{\beta^{2}}-\frac{\varphi_{2}}{\beta k p_{z}}\right)+\frac{\left(p_{z}-p_{2}\right)^{3}}{p_{z} \beta^{2}} \cdot\left(\frac{2}{3}-\frac{1}{3} k\right)-\frac{(k-1) Q_{3}}{A k p_{z} a} ; \\
Q_{3}=\frac{H_{u}}{H_{0} \alpha} .
\end{gathered}
$$

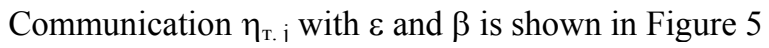

Cycle analysis for $\varepsilon=15$ and $\alpha=1,5$ shows (fig. 6 , a), what with $\beta=0.3-1.2 \mathrm{MPa}, 1^{0}$ it is possible to obtain almost the same efficiency values by changing the moment of the beginning of the heat supply $\varphi_{2}$, which is mainly determined by the value $p_{z}$. So, in the range $p_{z}=6.0-10 \mathrm{MPa}$, decrease by $1 \mathrm{MPa}$ causes a decrease in $\eta_{\text {т. } \mathrm{j}}$ by $1 \%$. 


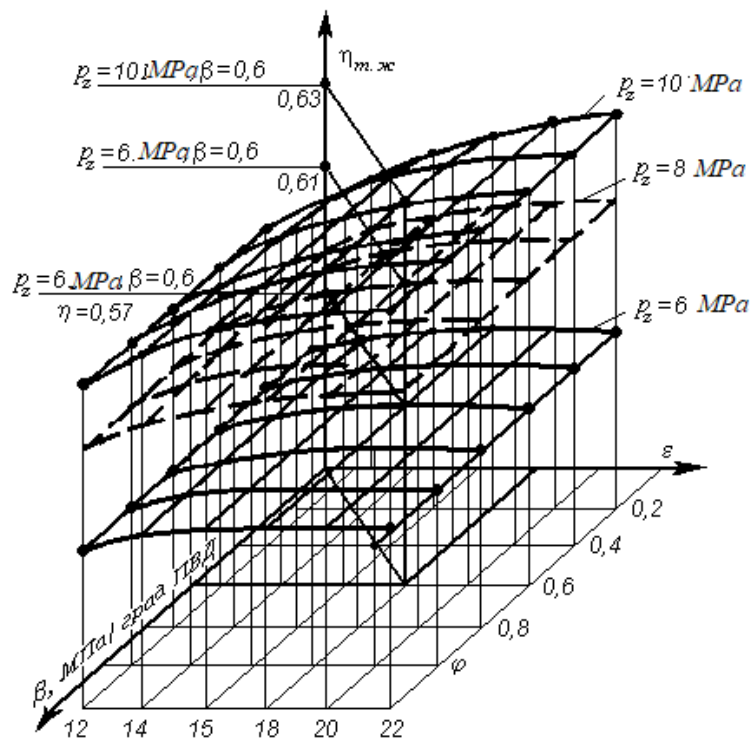

Fig.5. Dependences of the efficiency of the cycle on $\varepsilon$ and $\beta(\alpha=1.5)$ For high efficiency cycles with values

$\beta=0.4-1.2 \mathrm{MPa}, 1^{0}$ heat supply should begin $1-9^{0}$ before the TDC of the piston, which corresponds to the real conditions of the process. Carrying out a cycle with a pressure rise rate $\beta<0.3-0.4 \mathrm{MPa}, 1^{0}$ leads (all other things being equal) to a certain deterioration in economic indicators. The duration of the heat supply in the cycle $\varphi_{0}$ depends on and $p_{z}$ increases from $27-38^{0}$ at $\beta=12$ to $40-45^{\circ}$ at $\beta=0.2 \mathrm{MPa}, 1^{0}$.

It follows from the graphs (see Figure 6, b) [10] that the lower the rate of pressure rise in the cycle, the less intensively the initial period of heat release should proceed and the higher the heat release rate should occur in the final stage. The higher $p_{z}$, the more pronounced the indicated tendency.

Communication $\eta_{\text {т.j. }}$. with dynamic factor $\mathrm{D}$ is shown in Figure 7 , where the contours $\beta$ and $p_{z}$ are plotted, corresponding to the onset of fuel ignition in a real diesel engine. It follows from the graph that a small area can be outlined (shaded in the graph), which is limited by the curve $\varphi_{\text {н.в }}=12^{0}$ (the maximum possible lead from the point of view of stable ignition of the mixture in naturally aspirated diesel engines) and isolines $p_{z}=7.5-8.5$ MPa, in which low values of $D=1.9-1.2$ can be obtained with high fuel efficiency $\left(\eta_{\text {т.j. }}\right.$. decreases in comparison with the maximum achievable in the diesel cycle by $1.5-3 \%$ ). In this case, $\beta=0.3-0.4 \mathrm{MPa}, 1^{0} \mathrm{PCV}$. For a supercharged diesel engine, where values of $p_{z}$ $=10 \mathrm{MPa}$ and more, required for long-term operation, are achieved, the values of $\mathrm{D}$ can be obtained at $\varphi_{n . v}=16-12^{0} \mathrm{LDPE}$. Thus, it is possible to implement a soft diesel operating cycle with a high efficiency value. 

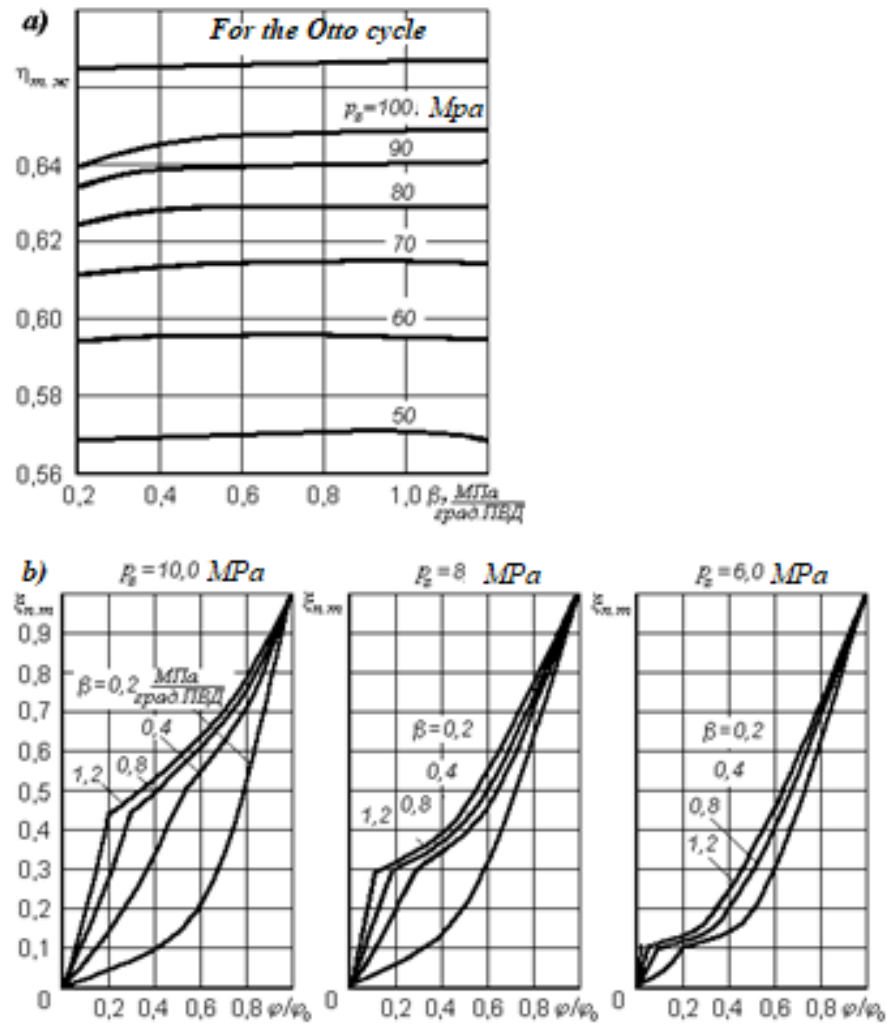

Fig.6. Dependences of the cycle parameters on $\beta$ and $p_{z,}$ a are efficiency coefficients $\eta_{m . j} ; \mathrm{b}$ are coefficients of fuel supply

The implementation of the required heat release in practice causes difficulties. With the generally accepted methods of conducting the working process, the opposite picture is observed: in the first period, heat release proceeds at high speeds, and in the final stage, the combustion rate and, accordingly, the heat release rate decrease. Therefore, the regularities of the heat supply obtained in the cycle analysis should be taken as a model to which one should strive when implementing special measures for organizing fuel supply and mixture formation in a real diesel engine.

Requirements for mixing processes are most simple to carry out efficient combustion of fuel in homogeneous and homogeneous mixtures. In a diesel engine, this is unacceptable, since at $\alpha \approx 1$, due to multistage ignition, due to the low octane number of diesel fuel, explosive combustion will occur, with high heat release rates. Meanwhile, with a separate supply of air and fuel to the cylinder, it is practically impossible to achieve even the permissible one, based on the dynamics of combustion, the homogeneity of the mixture, and in the diesel cylinder, there is always a separation of the charge. Implementing the optimal heat release process determines two main tasks that should be solved when organizing the mixture formation process in a high-speed diesel engine.

Firstly, to ensure the admissible degree of severity of the combustion process, it is necessary that the ignition starts as a little combustible mixture as possible in the diesel cylinder, corresponding in its concentration and temperature characteristics to the conditions of autoignition. The permissible minimum dose of a mixture capable of flammability is determined by the conditions for a stable process flow and ensuring an optimal rate of heat release. 
Secondly, for efficient combustion to occur, it follows that after ignition and the end of the period of rapid combustion, the fuel is evaporated and its vapors mixed with air at the highest possible rate. The process must be organized in such a way that the amount of oxygen necessary for its complete combustion is supplied to each fuel element, and the combustion products are removed from the combustion zone.

The known methods of mixture formation in diesel engines can be divided into two main groups: the first should include the schemes of chambers in which fuel is supplied to relatively stationary air; to the second - cameras, in

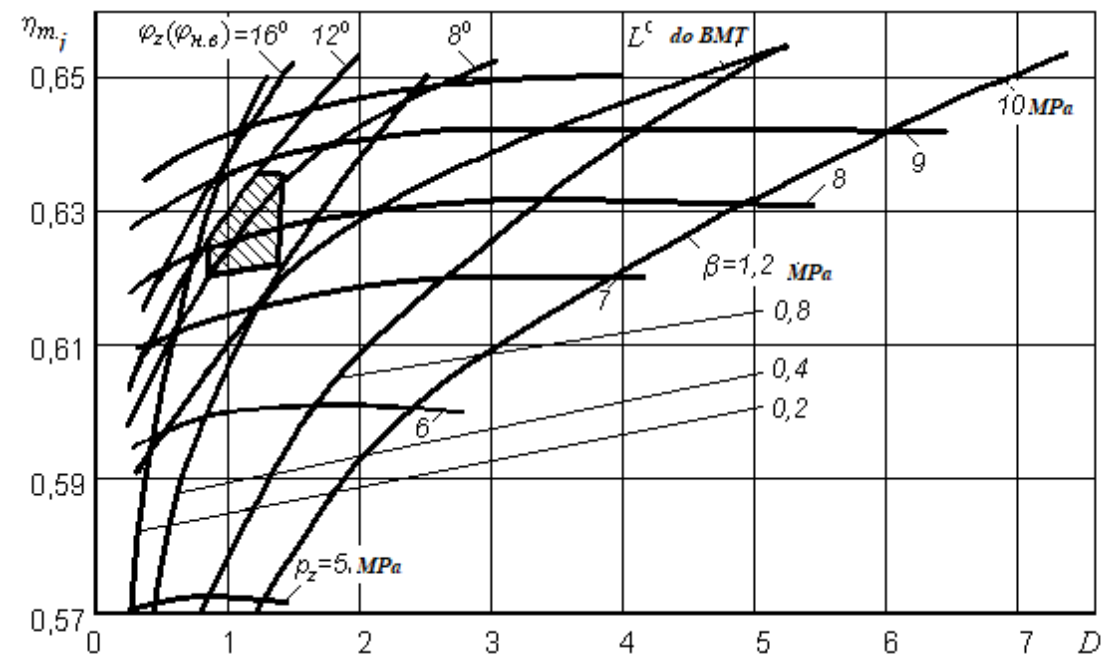

Fig.7. Dependence $\eta_{t . j}$. well, from the dynamic factor

Which air is supplied to the fuel to implement these methods, two types of combustion chambers are used: separated, in which fuel is injected into a volume isolated from the main space of the chamber, connected to the diesel cylinder by a relatively narrow channel; undivided with direct fuel injection into the over-piston space of the diesel cylinder.

According to the principle of mixture formation, one should distinguish between volumetric (jet) and wall (film) mixture formation chambers. Intermediate types of chambers are possible, in which elements of the volumetric and near-wall mechanisms of mixture formation coexist, for example, a CRDI-type chamber [11]. In each of these types of chambers, various means are used to solve the issues of ensuring a soft and economical combustion process.

The divided chambers are divided into vortex and pre-chambers. The first ones provide for blowing fuel jets with an air stream, and the second ones relate to a group of chambers in which fuel is supplied to the air. The main advantage of the separated chambers is the reduction of dynamic loads on the parts of the crank mechanism due to fuel ignition and a period of rapid combustion in a volume isolated from the above-piston space by a throat with a small flow area.

Separated chambers allow for a fairly high level of forcing diesel engines for $\mathrm{p}_{\mathrm{e}}$ and $\mathrm{p}_{\mathrm{d}}$, have a relatively low sensitivity to the quality of the fuel used and less toxicity of exhaust gases, especially due to the lower content of nitrogen oxides. However, engines with these chambers are characterized by increased not only specific fuel consumption due to gasdynamic energy losses when the charge flows through the throat from the chamber to the diesel cylinder, as well as increased heat losses through the developed chamber surfaces, but also by poor starting properties and a relatively complex head design. 
The divided chambers are small in size and do not require a long range of jets. For mixture formation in them, the gas-dynamic energy of charge flow during the compression stroke, as well as the energy of gases flowing out of the prechamber through the throat after fuel ignition is used; therefore, no special requirements are imposed on the fuel supply process in these chambers. In diesel engines with these chambers, pin sprays with a large flow area are most often used, providing a small value of $\varphi_{\mathrm{v}}$ even at high $\mathrm{p}_{\mathrm{d}}$. Required $p_{v}^{\max }=20-40 \mathrm{MPa}$. Unseparated chambers make it possible to obtain low specific fuel consumption in a wide range of operating modes of transport engines. There are two main groups of unseparated combustion chambers: open and semi-open types, although intermediate options are also possible. In open chambers, their cross-section with the largest area is in direct contact with the above-piston space and is close in size to the crosssectional area of the piston. In this case, the formation of the mixture occurs mainly in the air volume of the chamber (for example, the Gesselmann chamber).

In semi-open chambers, the cross-sectional area connecting the chamber with the abovepiston space is significantly less than the piston cross-sectional area (the throat or the diameter of the chamber itself is significantly less than the piston diameter). In chambers of this type, along with elements of the volumetric mixture formation scheme, elements of the near-wall scheme are manifested to one degree or another. These cameras are most common in ADT (see Figure 2). In some chambers, to ensure the required flow area of the inlet channels and valves and the removal of the nozzle from under the head cover, the nozzle is displaced and positioned obliquely relative to the cylinder axis.

In diesel engines with unseparated combustion chambers, multi-hole nozzles are used, forming a series of jets of atomized fuel interacting with an air charge moving in the combustion chamber.

The movement of air in the diesel cylinder is achieved by installing screens on the valves corresponding to the direction of the channels of the purge ports in two-stroke engines and the use of profiled intake channels of tangential and snail types. The intensity of the tangential vortex formed in these chambers in modern diesel engines is very high - air velocities at a distance of several millimeters from the chamber wall are equal to $15-40 \mathrm{~m}$, $\mathrm{s}$, and in some models reaches high values [11].

In modern diesel engines, volumetric chambers have become widespread, in which a beneficial effect on the working process can be carried out very effectively by controlling the fuel injection. In these chambers, to ensure rapid burnout of the bulk of the fuel in periods III, IV, it is necessary to distribute the fuel as evenly as possible in the chamber space (macro-mixing), as well as to homogenize the charge and increase the homogeneity of the mixture (micro-mixing).

From the point of view of macro-mixing requirements, it is necessary to create a certain spatial orientation of the jets, taking into account the shape of the chamber and the direction of movement of the air charge. Homogenization and homogeneity of the mixture are achieved by feeding the fuel into the chamber in a finely atomized form. An excessive reduction in the diameter of the droplets leads to a rapid decay of their velocity, inhibits the evaporation of fuel, and worsens the distribution of vapors over the chamber volume.

Modern fuel systems provide droplets with a size of 10 - 30 microns or less in the main injection phase. The size of droplets in the jet in different phases is not the same. Due to the low injection pressures, very large droplets (up to 200 microns) can form in the initial and final periods. The presence of large droplets in the final phase of injection is highly undesirable since this can lead to incomplete combustion of fuel, accompanied by an increase in fuel consumption and smoke.

In volumetric chambers, the tangential vortex is predominant, which, blowing the jets from the side surface, promotes intensive heating and evaporation of droplets, mixing of fuel vapors with air and, as the concentration of the fuel-air mixture favorable for 
combustion is created, the rapid and complete fuel burnout. The fuel vaporization in the frontal zone will occur efficiently as long as the fuel meets a hot air charge on its way. When the jet reaches the relatively cold chamber wall, the evaporation rate drops sharply [12].

In advanced adiabatic diesel engines, the combustion chamber walls have a higher temperature. However, in this case, the ingress of fuel onto the chamber walls is undesirable since it cracks with the release of free carbon. With a decrease in the length of the jet in comparison with the distance of its free development to the chamber wall, there is an underutilization of the air charge and a decrease in the period of intensive evaporation of the fuel, which will also reduce the efficiency of mixture formation.

Suppose the induction period is less than the time the jet reaches the wall. In that case, the transport of energy by drops to the frontal zone of the jet is disturbed because it is passing through the flame zone, the drops evaporate, the formation and advancement of the front are inhibited, which leads to a reduction in the jet length in comparison with its effective value.

Suppose the induction period exceeds the time the jet reaches the wall $\left(\tau_{\mathrm{cT}}\right)$. In that case, this leads to the formation of many fuel vapors in the chamber and their simultaneous burnout during the period of rapid combustion and, accordingly, will lead to hard operation of the diesel. Thus, the optimal conditions are those under which $\tau_{\mathrm{i}}$ is close in its value to the duration of the jet front reaching the wall $\left(\tau_{\text {ст }}\right)$, and the range of the jets as close as possible to their free movement to the chamber wall $[13,14]$.

With significant deviations from the above scheme, deterioration in combustion efficiency should be expected (Figures 1-9). Under partial load conditions, when there is a significant excess of air, and the range of the jets is less than the path of their free development to the wall, they should propagate by the time of the onset of ignition by an amount close to their maximum range.

In the above model of mixture formation, preference is given to elements that organize macro-mixture formation. The main factor for this is also the fact that at $p_{v}^{\text {max }}$ exceeding 20-25 MPa, the total atomization fineness, characterized by the average droplet diameters, changes insignificantly [15]. In addition, according to CRDI experiments, when injecting a fuel-air mixture, despite finer atomization of fuel, the economic performance of a highspeed diesel engine turns out to be worse, and the smoke of the exhaust gases is higher than with conventional injection, which can only be explained by a decrease in the jet range. The stated conditions for the organization of effective mixture formation are also confirmed by the fact that the injection advance angles corresponding to $g_{e}^{\min }$ during the operation of the TA with different volumetric fuel delivery rates have values at which the jets reach the walls of the combustion chamber at the moment close to the start of fuel ignition. 

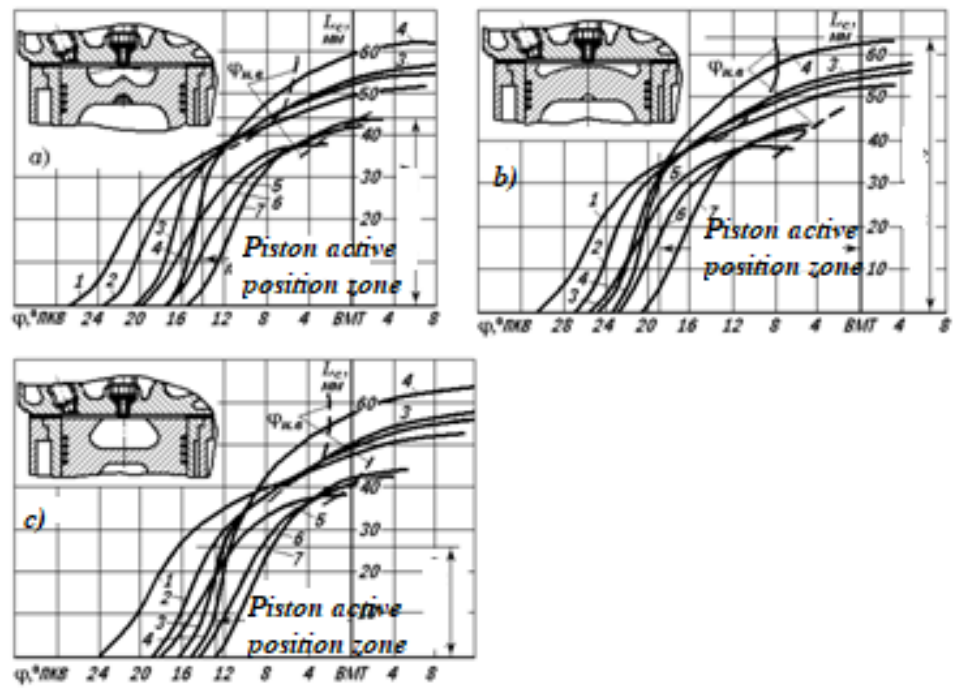

Fig.8. the nature of the development of the range of fuel jets, relative to the position of the piston to TDC, depending on the type of combustion chamber, a is YMZ; b is Gesselman; $\mathrm{c}$ is CRDI; at $p_{v}^{\text {max }}$, respectively, for $\mathrm{V}_{\mathrm{c}}$ of $160 \mathrm{~mm}^{3}: 1$ of $26 \mathrm{MPa} ; 2$ of 36,4 MPa; 3 of $44 \mathrm{MPa} ; 4$ of $51,2 \mathrm{MPa}$; for $\mathrm{V}_{\mathrm{c}}$ of $40 \mathrm{~mm}^{3}: 5$ of $10.0 \mathrm{MPa}$; 6 of $13 \mathrm{MPa} ; 7$ of $16 \mathrm{MPa}$; (diesel AM-74, S,D $=150,160 \mathrm{~mm}, \mathrm{n}_{\mathrm{d}}=1500$ $\min ^{-1}, \mathrm{~V}_{\mathrm{c} . \mathrm{n}}=160 \mathrm{~mm}^{3}$ )

With an increased diameter of the chamber with a large predominance of elements of the volumetric mixture formation scheme (Gesselman chamber), the fuel jets at extreme lead angles do not reach the chamber walls since the range of the jets with the implemented TA variants is insufficient. An increase in the advance angle could, in this case, also slightly increase the degree of penetration of the jets into the chamber by the time the ignition starts; however, this pushes the onset of fuel ignition from TDC and without this earlier than in the Leyland-YaMZ chamber, which leads, due to the lower degree of expansion to increase $g_{e}$.

In the CRDI chamber, the conditions are optimal under which the fuel starts to ignite after the jets reach the chamber wall. This result is associated with the need to form a wall layer in a chamber of this type by the time of ignition. Confirmation of the stated conditions for optimizing mixture formation is the results that were obtained during the development of the TA parameters for diesel engines A90TK (Figure 9) [16]. The smallest specific fuel consumption is observed when the time the jet reaches the chamber wall $\phi_{s t}$ approaches the duration of the induction period $\varphi_{\tau_{i}}$. 


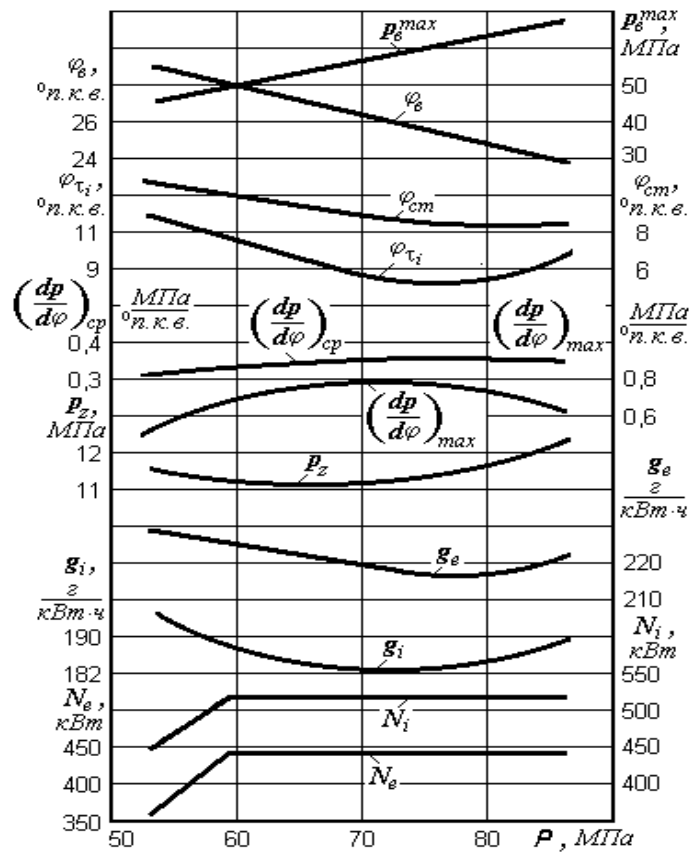

Fig. 9. Dependencies of indicators and parameters characterizing the processes

In undivided chambers with a predominance of elements of the film mixture formation scheme (types of Meurer, Pischschinger), the fuel is applied in the form of a film to the chamber surface using a double or single-hole atomizer, where it evaporates and burns, reacting with air oxygen moving relative to this surface. Due to the intensive rotational movement of the charge, the lighter combustion products are squeezed out to the center of the chamber, and the cold and heavy air charge of mixture formation from the criterion of the injection intensity enters the surface of the chamber due to which it is used very effectively and contributes to the achievement of high values $p_{i}$.

In chambers of the CRDI type, fuel is applied to the wall in the form of a droplet film structure, and its evaporation occurs mainly when heated from the hot wall of the chamber, and mixture formation is carried out due to intensified radial vortices. These chambers require a sufficiently accurate supply of fuel to specific areas in order to efficiently vaporize it.

The advantage of chambers in which elements of the near-wall mixture formation prevail is that they allow limiting the rate of heat release during the period of rapid combustion at sufficiently high economic indicators. This is due to the fact that in the initial period of the combustion process, only an insignificant part of the injected dose of fuel is involved in the formation of the combustible mixture, and the main amount is preserved in the wall layer in the form of a film on the walls of the chamber.

There are three known methods for reducing the induction period: increasing the temperature and pressure of the air charge, increasing the cetane number of the fuel, and controlled fuel supply. In naturally aspirated diesel engines, it is possible to increase the temperature and pressure slightly by increasing the compression ratio (increasing the compression ratio from 15 to 18 leads to a decrease in the induction period by only $10 \%$ ). However, increasing the compression ratio requires special measures in serial production to stabilize the value of the over-piston clearance, which increases the cost of manufacturing engines. This is more efficiently implemented in supercharged diesel engines, which are 
becoming more widespread and where, due to a decrease in the induction period due to higher temperatures at the end of charge compression, it is possible to reduce the maximum degree of pressure rise in the diesel cylinder by 1.5-2.0 times. Obviously, a decrease in the induction period will also be in adiabatic diesel engines.

A significant increase in the cetane numbers of the fuel with the used petroleum feedstock and technological processes of its processing did not find industrial implementation. Studies with special additives have confirmed their effectiveness in reducing the induction period. However, most of these additives are poisonous or highly volatile and therefore do not find use. It should also be borne in mind that a significant reduction in the induction period can lead to a deterioration in the economic performance of a diesel engine due to a decrease in the time allotted for the processes of mixture formation.

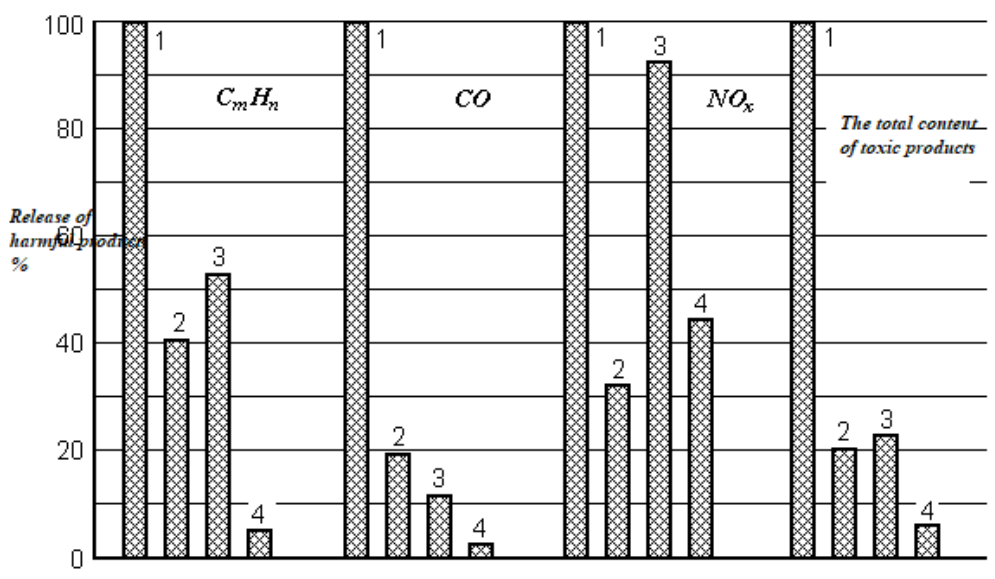

Fig. 10. Comparison of toxicity of different types of engines: 1 is gasoline (taken as 100\%); 2 are spark ignition engines running on liquefied gas; 3 are diesel engines with direct fuel injection; 4 are diesel engines with pre-chamber mixture formation

Influence of processes of mixture formation on toxicity and opacity of exhaust gases. The composition of harmful components emitted with exhaust gases depends on the type of engine (Fig. 10) and its operating modes (Figure 11). In ATD, the harmful components are fine solid particles of carbon - soot, which determines the smoke of exhaust gases, and gaseous products. The main gaseous toxic components in diesel exhaust gases are nitrogen oxides $\left(\Sigma \mathrm{NO}_{\mathrm{x}}\right)$, carbon monoxide $(\mathrm{CO})$, as well as unburned hydrocarbons $\left(\mathrm{C}_{\mathrm{m}} \mathrm{H}_{\mathrm{n}}\right)$ and aldehydes. The approximate relationship of the dimensions of the quantities in which the content of harmful emissions in the exhaust gases is estimated is presented in Table 1.

For the overall assessment of toxic substances, the concentration index $\mathrm{K}_{\Sigma}$ is also used, which is a dimensionless value equal to the multiplicity of such a dilution of exhaust gases with air, at which the concentration of each of the components under consideration (CO, $\mathrm{NO}_{\mathrm{x}}, \mathrm{C}_{\mathrm{m}} \mathrm{H}_{\mathrm{n}}$ ) would equal its maximum permissible daily average concentration (MPC) in atmospheric air of settlements.

$$
\mathrm{K}_{\Sigma}=\mathrm{CO},|\mathrm{CO}|+\Sigma \mathrm{NO}_{\mathrm{x}}\left|\Sigma \mathrm{NO}_{\mathrm{x}}\right|+\mathrm{C}_{\mathrm{m}} \mathrm{H}_{\mathrm{n}}, \mathrm{C}_{\mathrm{m}} \mathrm{H}_{\mathrm{n}} \mid
$$

Where $\mathrm{CO}, \Sigma \mathrm{NO}_{\mathrm{x}}, \mathrm{C}_{\mathrm{T}} \mathrm{H}_{\mathrm{n}}$ is concentration of toxic components in exhaust gases; $|\mathrm{CO}|$, $\left|\Sigma \mathrm{NO}_{\mathrm{x}}\right|,\left|\mathrm{C}_{\mathrm{m}} \mathrm{H}_{\mathrm{n}}\right|-\mathrm{MPC}$

According to the existing technical standards, the average daily MPC in the atmospheric air have the following values: $|\Sigma \mathrm{CO}|=0.0006 \mathrm{mg}, \mathrm{m}^{3},\left|\Sigma \mathrm{NO}_{\mathrm{x}}\right|=0,0085 \mathrm{mg}, \mathrm{m}^{3}$. The most harmful products are nitrogen oxides, the content of which in exhaust gases can reach - 
$0.5 \%$ by volume and which mainly determine the toxicity of emissions in diesel engines. The CO content in diesel engines, in contrast to gasoline engines, is low and does not exceed $0.5 \%$. The emission of hydrocarbons (in terms of methane) can reach about $0.5 \%$, and aldehydes (in terms of formaldehyde) - about $0.002 \%$.

Table 1 . Conditional conversion of units of measurement of parameters of toxic constituents of exhaust gases

\begin{tabular}{|c|c|c|l|}
\hline $\mathrm{CO}$ & $\mathrm{C}_{\mathrm{T}} \mathrm{H}_{\mathrm{p}}$ & $\Sigma \mathrm{NO}_{\mathrm{x}}$ & unit of measurement \\
\hline 1.00 & 1.00 & 4.0 & $\mathrm{mgk}, \mathrm{DJ}$ \\
3.65 & 3.65 & 13.6 & $\mathrm{~g},(\mathrm{~kW} \cdot \mathrm{h})$ \\
0.68 & 0.68 & 2.8 & $\mathrm{mg}, 1$ \\
600 & 1300 & 1400 & chnm (PPM) \\
0.06 & 0.13 & 0.14 & \% (by volume) \\
\hline \multicolumn{4}{|c|}{ When recalculating. it is accepted: $\mathrm{g}_{\mathrm{e}}=272 \mathrm{~g},(\mathrm{~kW} \cdot \mathrm{h}) ; \alpha=1.6}$. \\
\hline
\end{tabular}

The main component of nitrogen oxides in exhaust gases is nitrogen oxide NO (up to $90 \%$ ). which. when released into the environment. is rapidly oxidized to nitrogen dioxide $\mathrm{NO}_{2}$. Average values of toxic components in diesel engines of various types are presented in table 2.

Table 2. Limit values for the content of toxic products in diesel engines

\begin{tabular}{|c|l|c|c|c|}
\hline $\begin{array}{c}\text { Frequency } \\
\text { mode }\end{array}$ & $\begin{array}{c}\text { Limits of } \\
\text { change }\end{array}$ & $\begin{array}{c}\Sigma \mathrm{NO}_{\mathrm{x}} . \\
\mathrm{mg}, 1\end{array}$ & $\mathrm{C}_{\mathrm{t}} \mathrm{H}_{\mathrm{p}}$ & $\begin{array}{c}\mathrm{CO} . \\
\%\end{array}$ \\
\hline $\mathrm{P}_{\mathrm{D} . \mathrm{N}}$ & Upper & $3-4.5$ & $0.70-0.90$ & $0.12-0.18$ \\
\hline $\mathrm{P}_{\mathrm{D} . \mathrm{N}}$ & Lower & 2 & $0.06-0.25$ & $0.13-0.11$ \\
\hline $\mathrm{P}_{\mathrm{D} . \mathrm{M}}$ & Upper & 6 & $0.80-0.50$ & $0.07-020$ \\
\hline $\mathrm{P}_{\mathrm{D} . \mathrm{M}}$ & Lower & 2 & $0.05-0.25$ & $0.02-0.10$ \\
\hline
\end{tabular}

The lower limits of changes in toxic components refer to diesel engines with prechamber and vortex-chamber mixture formation. the upper ones - to diesel engines with direct fuel injection. Diesels with film (wall) mixture formation (M-process) differ little from diesel engines with a volumetric mixture formation in terms of the content of toxic components. The average values of the toxicity level of some domestic diesel engines with direct fuel injection. determined according to OST 23.1.440-76. are presented in table 3.

Table 3. Toxicity level of Russian ATD

\begin{tabular}{|l|c|c|c|c|c|c|c|c|c|}
\hline \multicolumn{2}{|c|}{ Diesel model } & $\begin{array}{c}\text { D- } \\
144\end{array}$ & $\begin{array}{c}\text { D- } \\
120\end{array}$ & $\begin{array}{c}\text { SMD-- } \\
18 \mathrm{H}\end{array}$ & $\begin{array}{c}\text { SMD-- } \\
66\end{array}$ & $\begin{array}{c}\text { D- } \\
240 \mathrm{~T}\end{array}$ & $\begin{array}{c}\text { SMD-- } \\
31 \mathrm{~A}\end{array}$ & $\begin{array}{c}\text { YaMZ } \\
-238\end{array}$ & $\begin{array}{c}\text { YaMZ } \\
-740\end{array}$ \\
\hline Emission. & $\mathrm{N} \mathrm{NO}_{\mathrm{x}}$ & 17.8 & 14.3 & 22.0 & 17.3 & 16.0 & 13.8 & 16.9 & 19.0 \\
\cline { 2 - 10 } $\mathrm{g},(\mathrm{kWh})$ & $\mathrm{CO}$ & 6.5 & 1.8 & 7.3 & 3.8 & 2.2 & 4.3 & 6.0 & 4.0 \\
\hline
\end{tabular}

In recent years, work has intensified to improve the processes of mixture formation in diesel engines with direct fuel injection by developing new, more advanced chamber schemes. The widespread introduction of pressurization, in which there is a high excess air ratio, contributes to reducing smoke during operation under steady-state conditions. A significant effect on the content of toxic constituents in the exhaust gases can be exerted in diesel engines with direct injection by controlling the fuel supply process. 

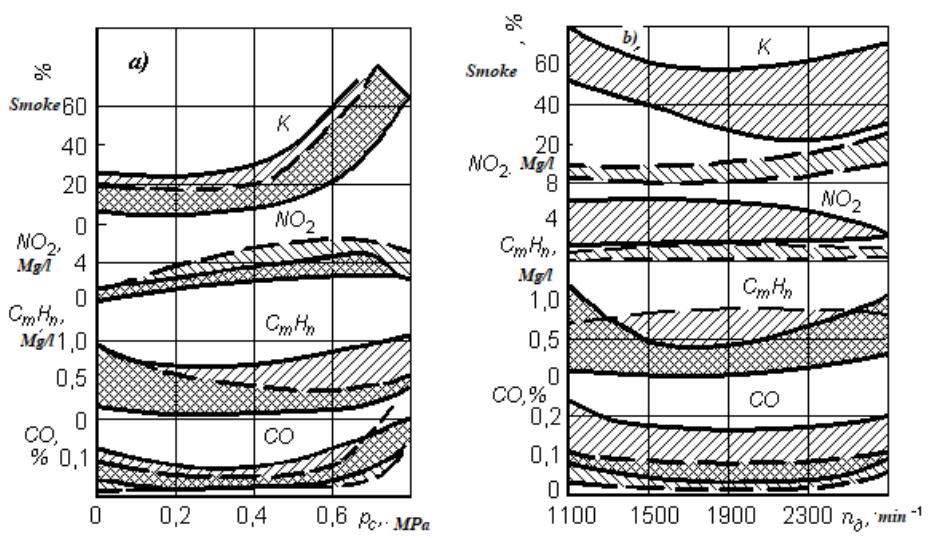

Fig.11. Change in the content of harmful components in the exhaust gases of diesel engines (with direct injection): $\mathrm{a}$ - according to the load characteristic; $\mathrm{b}$ - according to the speed characteristic; speed characteristic; mode - - idle

\section{Conclusion}

Analysis of the existing theoretical provisions regarding the formalization of the combustion process revealed the presence of three fundamentally different schemes [8. 13. 17. 18. 19. 20 and 21]:

The first (Semenov N.N.. Vibe I.I.. MSTU named after N.E. Bauman. etc.) interprets it as a two-phase, including kinetic and diffusion;

The second (CRDI. N.I. Ivanchenko. etc.) asserts the presence of four phases: coldflame, fast, main and delayed;

The third (CRAT. Sviridov Yu.B.. Fainleib B.N. and others), without challenging the CRDI scheme, additionally establishes a controlled combustion phase between the fast and main combustion phases.

We have adopted the third scheme, but the first is not refuted either. The only difference is that we assume the probability of kinetic and diffusion reactions occurring in any of the five phases of the process.

Analysis of the results of previously performed studies shows that the design of the combustion chamber significantly affects the nature of mixture formation and the duration of each phase. The CRDI-type chamber, in comparison with the Meurer and Gesselman chambers, provides the onset of ignition closer to the TDC, does not require a jet range and has the smallest zone of the active position of the piston. These properties, although they limit the possibilities of forcing diesel engines of the CS CRDI in terms of speed, are very attractive from the point of view of partial adiabatization of the cycle.

Analysis of the existing methods and criteria for assessing the effect of thermal insulation of the combustion chamber elements on the nature of the process indicates a lack of consensus and efficiency indicators of changes made to diesel engines. The estimates of CRDI have some mathematical and physical inaccuracies.

\section{References}

1. Tolstov A.I. Ignition lag indicator period and dynamics of the cycle of a high-speed engine with compression ignition. Tr., NILDa. (1), pp. 5-55, (1955)

2. Iost V. Explosions and combustion in gases. Publishing house of foreign, Moscow, p. 
688, (1952)

3. Ricardo G.R. High-speed internal combustion engines. Moscow: Mashgiz. p.409. (1960)

4. Brose D.D. Combustion in piston engines. Moscow: Mechanical Engineering. p. 247, (1969)

5. Semenov N.N. On some problems of chemical kinetics and reactivity. Moscow: Publishing house of the Academy of Sciences of the USSR. p. 350, (1954)

6. Sh Brose D.D. Combustion in piston engines, Moscow, Mechanical Engineering, p. 247, (1969)

7. Tern V.Ya. Mechanism of hydrocarbon oxidation in the gas phase, Moscow, Publishing house of the Academy of Sciences of the USSR. p. 496, (1960)

8. Sokolik A.S. Self-ignition. flame and detonation in gases. Moscow: Publishing house of the Academy of Sciences of the USSR. p. 425, (1960)

9. Ivaschenko N.A., Petrukhin N.V. Method of joint modeling of the working process and thermal state of the CPG of the "adiabatic engine", Mechanical engineering, (2). pp. 61-65, (1987)

10. Fainleib B.N. Kulikov V.P. Investigation of the relationship between vibrations of the piston ring of a YaMZ-236 diesel engine and the rigidity of the combustion process, Proc. CRAT, 70. pp. 20-26, (1977)

11. Fainleib B.N. Analysis of the thermodynamic cycle of high-speed engines with compression ignition. Tr. CRDI. 25, pp. 29-38, (1965)

12. Ivanchenko N.N. Semenov B.N. Sokolov V.S. Working process of diesel engines with a chamber in the piston, Mechanical Engineering. p. 228, (1972)

13. Nikitin M.D. Kulik A.Y. Zakharov N.I. Heat-protective and wear-resistant coatings of diesel engine parts, Mechanical engineering. p. 168, (1977)

14. Fainleib B.N. Baraev V.I. Investigation of the influence of the injection pressure level on the parameters of the working process of a high-speed dieel, Tractors and agricultural machines, (4), pp. 10-12, (1971)

15. Fainleib B.N. Baraev V.I. Increasing the efficiency of mixture formation in diesel engines by influencing the dynamics of a sprayed jet of fuel, Dvigatelestroyeniye, (9), pp. 8-12, (1986)

16. Brief description and operating instructions for the MAI-2A electro-pneumatic stroboscopic indicator. Moscow Aviation Institute, Moscow, (1973)

17. Fainleib B. N. Shatalov G.S. Substantiation of the parameters of the fuel equipment of the forced diesel engines ChN. 16.5, 17 for heavy industrial tractors, Dvigatelestroyeniye, (7), p. 912, (1986)

18. Aripdjanov M.M. Ismatov J.F. Technology of use and production of technical ceramics in internal combustion engines, Composite materials. UzR. Scientifictechnical. and practical journal, 2. pp. 27-29, Tashkent, (2003)

19. Vibe I.I. New about engine duty cycle. p. 271. Moscow, (1962)

20. Kavtaradze R.Z. Local heat exchange in piston engines, Publishing house of MSTU im. N.E. Bauman. p. 592, (2001)

21. Kadyrov S.M., Aripdzhanov M.M., Ismatov J.F. Indicators of a diesel engine with ceramic parts. Abstracts of the XXXIX International Scientific and Technical Conference. Moscow: MSTU MAMI, pp. 21, (2002)

22. Sviridov Y. B. Mixture formation and combustion in diesel engines. - L: Mechanical engineering, 1972, p. 224. (1972)

23. Tolstov. A.I. Ignition lag indicator period and dynamics of the cycle of a high-speed engine with compression ignition. Tr. NILDa, pp. 5-55, (1955) 